\title{
Dynamic monitoring of the driving wheels' pre-sliding of the railway transport
}

\author{
Mikhail Chuveyko ${ }^{1, *}$, Sergey Nosachev ${ }^{1}$ \\ ${ }^{1}$ Don State Technical University, Automation of Production Processes Department, 344000, Gagarin sq. 1, Rostov-on-Don, Russia
}

\begin{abstract}
The proposed method of dynamic monitoring of the driving wheels' state of the railway transport makes it possible to detect in a timely manner the processes accompanying the transition of the wheel from the adhesion to slipping mode. This in turn helps prevent a loss of adhesion traction that have a wide range of negative consequences. The method is based on the spectral analysis of the vibration processes of the driving wheels of the railway transport.
\end{abstract}

\section{Introduction}

The task of identifying and preventing loss of traction appeared in the same period when the first self-propelled wheeled vehicles were created. Due to the continuous increase in the power of the railway transport drives, the likelihood of loss of traction increases, and therefore this problem is most actual in railway transport. Despite the deceptive simplicity, the problem of adhesion the wheel with the road surface is one of the oldest and most complex problems of wheeled vehicles $[1,2]$.

\section{The model description}

To solve this problem, consider the properties of the subsystem "wheel-rail". In contrast to the classical simplified concepts of the transformation of rotational motion into translational motion, the speed of rolling of the wheel is determined not only by its angular frequency and radius, but also by the deformation processes occurring in the wheel. This phenomenon is known as elastic sliding or "creep". It was considered in more detail in [3-8]. This phenomenon leads to the fact that the actual speed of the wheel during the action of the external load and in the absence of loss of adhesion is less than its ideal value (determined by the formula $\omega R$ ). The difference between the actual and ideal velocities determines the speed of elastic sliding. Experiments show that the speed of elastic sliding depends on many factors, including external tangential $F_{\tau}$ and normal $F_{n}$ loads on the wheel [9-12]. A mathematical model describing the dynamic processes taking place in the subsystem "wheel-rail" was considered in [6]. Let's consider it in more detail.

In constructing the model, the wheel was considered as an elastic-dissipative rim interacting with the wheel axis by means of an elastic-dissipative coupling formed by the disk. The concept of building a model is based on the use of the principle of flows and accumulators, which makes it possible to greatly simplify the analysis of the dynamics of the system. Simplification is achieved by using the division of the simulated wheel into small stationary (relative to the rail) sectors. Thus, the variables characterizing the state of the wheel in individual sectors are "tied" not to specific moving wheel points, but to the geometric position of these sectors relative to the contact spot. While its movement, the material forming the rim of the wheel rotates around the wheel axis. This movement leads to the fact that through the boundaries of each such stationary sector passes a certain amount of mass of the rim of the wheel. To describe the velocity of the material passing through the sector boundary, we will use the value of the "material flow" $S[\mathrm{~kg} / \mathrm{s}]$. In the absence of deformation of the rim, the total mass of the incoming (with a positive sign) and the outgoing (with a negative sign) material will be zero. The flow in the absence of deformations and determined only by the angular frequency of rotation of the wheel axis will be denoted as $\bar{S}$. However, in the presence of deformations, the mass of material can accumulate within the sector. To numerically characterize the accumulation phenomenon, we introduce the notion of the coefficient of compaction of material $\tau$, which characterizes the increase in the density of the material in each individual sector. Deformations are a consequence of forces acting on the elements of the wheel rim. In the model, forces are distinguished: the elastic-dissipative interaction of the rim of the wheel and the disk, the elastic-dissipative interaction of individual elements of the wheel rim with each other and the forces formed in the zone of tribocontact of the wheel and rail.

To characterize the force influence of the disk on the wheel rim element, the coefficients of the specific tangential elasticity $k_{1}$ and the specific tangential dissipation $h_{1}$ of the disk are introduced. The coefficient $k_{1}$ characterizes the force reaction acting from the disk side to the element of the rim of the wheel of unit length

\footnotetext{
* Corresponding author: chuveyko@icloud.com
} 
when it is displaced relative to the undeformed state per unit length. This coefficient is determined by the design of the wheel and the material shear module. To describe the amount of shift of an individual element of the wheel rim with respect to its undeformed state, the so-called tension value $N[\mathrm{~kg}]$ is introduced. It determines the mass of the rim material that must pass through a fixed cross-section to neutralize the deformation. Thus, each section of the wheel is characterized by its value $N$.

To characterize the force effect of individual elements of the wheel rim, one introduces the coefficients of the specific longitudinal elasticity $k_{2}$ and the specific longitudinal dissipation $h_{2}$. The coefficient $k_{2}$ characterizes the force response of the sector of the rim of the wheel of a unit length when it is stretched per unit length. This coefficient is determined by the design of the rim of the wheel and the Young's modulus for the material. The coefficients $h_{1}$ and $h_{2}$ characterize the dissipative component of the reaction forces and determine the values of the forces arising in response to the change in the strain quantities.

Dynamic connection, formed by tribological processes of the contact zone of the wheel and rail, is the cumulative result of the interaction of individual elements of the wheel and rail. At the same time, various elements participating in the interaction can be in different modes of operation. According to modern ideas about the process of friction [13-16], we can conditionally distinguish two modes of operation: "adhesion mode" and " sliding mode":

The "adhesion mode" is characterized by the presence of physico-mechanical bonds between the elements of the interacting surfaces. These links prevent the relative motion of the surfaces, which mathematically can be expressed by means of the force function $\mathrm{F}$ in the state coordinates. Studies have shown that the form of the force function is identical to Hooke's law: $F=k_{3} \Delta x$.

"Sliding mode" is characterized by the absence of permanent physico-mechanical bonds between the contacting surfaces, while the surfaces are in constant motion relative to each other. The process of formation and destruction of new bonds leads to the appearance of an interaction force, which is called the sliding friction force. The magnitude of this force is determined by the formula: $F=k_{4} F_{n}$. This expression includes the value of the coefficient of friction $k_{4}$. The magnitude of the coefficient of friction is determined by the properties of the interacting surfaces, as well as by the speed of relative slip. As the relative slip speed decreases, the intensity of bond formation increases, while the intensity of their destruction decreases, and with a decrease in speed below the threshold value this process leads to the formation of stable bonds and the transition to the meshing regime. It is empirically found that the effect of velocity can be well approximated by the expression:

$$
k(v)=\operatorname{sign}(v)\left(1-\xi\left(1-e^{-\chi v}\right)\right)
$$

Taking all this into account, the mathematical model describing the dynamics of a moving wheel has the form:

$$
\left\{\begin{aligned}
\frac{d S}{d t} & =k_{2} \frac{\partial}{\partial l} \frac{1}{\tau}-h_{2} \frac{\partial}{\partial l}\left(\frac{1}{\tau^{2}} \frac{d \tau}{d t}\right)- \\
& -\frac{1}{H \bar{Q}}\left(k_{1} N+h_{1} \frac{d N}{d t}\right)+f(v) \\
\frac{d N}{d t} & =S-\bar{S} \\
\frac{d \tau}{d t} & =-\frac{1}{H \bar{Q}} \frac{\partial S}{\partial l} \\
\frac{d x}{d t} & =v \\
\frac{d v}{d t} & =\frac{1}{m}\left(\int_{D} f(v) d l-F_{n}\right)
\end{aligned}\right.
$$

where: $H$ is the area of the wheel $\operatorname{rim} ; \bar{Q}$ is the initial density of the rim material; $f(v)$ is the specific force of the contact interaction; $D$ is the extent of the contact spot; $x$ is horizontal coordinate of the wheel center; $v$ is translational velocity of the wheel center; $m$ is the mass of the wheel; $F_{n}$ is the force of external action.

\section{The method description}

Studies of this model have shown that as the "wheel-rail" system approaches the pre-sliding state, the dynamic properties of the contact change. This is due to changes occurring in the contact spot.
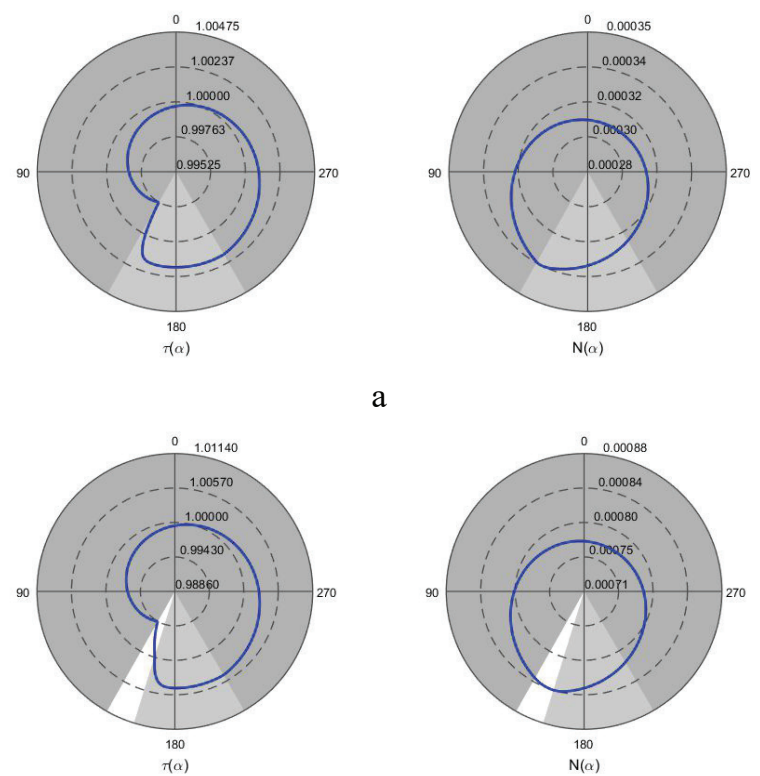

b
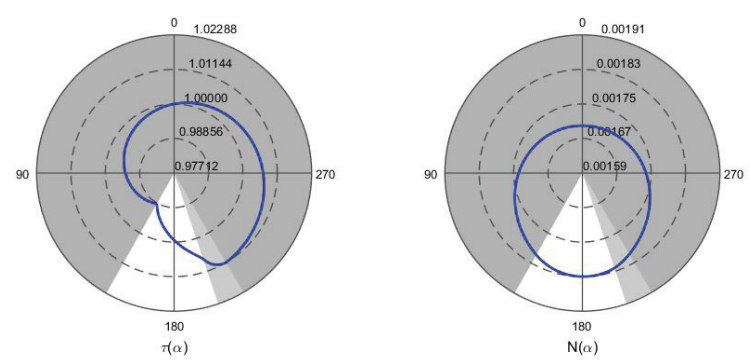

Fig. 1. The distribution of the coefficient $\tau$ and $N$ along the surface of the wheel with variations in the tangential load: a - $2 \mathrm{kN}, \mathrm{b}-5 \mathrm{kN}, \mathrm{c}-11 \mathrm{kN}$. 
In Figure 1 shows the diagrams of the dependence of the coefficient of compaction of the material $\tau$ and the value of the tension $N$ on the angle of the sector on the wheel. The Figure 1 shows the results of experiments for three different values of the tangential load and the following color scheme is applied: dark gray - the area of the wheel, which is outside direct contact with the rail; light gray - the zone of the wheel, which is in an elasticallyengaged interaction with the rail; white - the area of the wheel, which is in the sliding mode along the surface of the rail.

The diagrams in Fig. 1 it is easy to see that as the system approaches the pre-sliding state, a substantial change in the picture of the deformation distribution is observed, and, in view of the fundamental dependence of the elastic sliding effect on deformation processes, it is natural to expect a change in the properties of the system conjugate to this effect. As the system approaches the pre-sliding state, a change in the parameters of small oscillations of the wheel speed in response to external perturbations should be observed.

Considering the linearized model of the "wheel-rail" system (1) about some steady-state motion, it is possible to introduce transfer functions characterizing the influence of the external conditions $\left(F_{n}, F_{\tau}\right.$ and $\left.\omega\right)$ on the translational speed of the wheel:

$$
\left(\begin{array}{c}
v(p) \\
x_{2}(p)
\end{array}\right)=\left(\begin{array}{lll}
W_{v \tau} & W_{v n} & W_{v \omega} \\
W_{x \tau} & W_{x n} & W_{x w}
\end{array}\right)\left(\begin{array}{l}
F_{\tau}(p) \\
F_{n}(p) \\
\omega(p)
\end{array}\right)
$$

where: $F_{n}(p), F_{\tau}(p), \omega(p)$ are the Laplace transform corresponding to the deviations of the tangential force, normal force and angular frequency from its stationary values; $W_{i j}$ is transfer functions by the corresponding channels.

Of special interest from the point of view of diagnosis is the transfer function $\mathrm{W}_{v \tau}$, which describes the influence of the normal component of the external force on the speed of the wheel. Without going into all the properties of the given transfer function, we note the key property: in the low-frequency range (tens of hertz), this transfer function can be considered as a gain $k$. In addition, the value of this coefficient essentially depends on the stationary regime of motion. As the system approaches the pre-sliding state, $k$ increases significantly (see Figure 2).

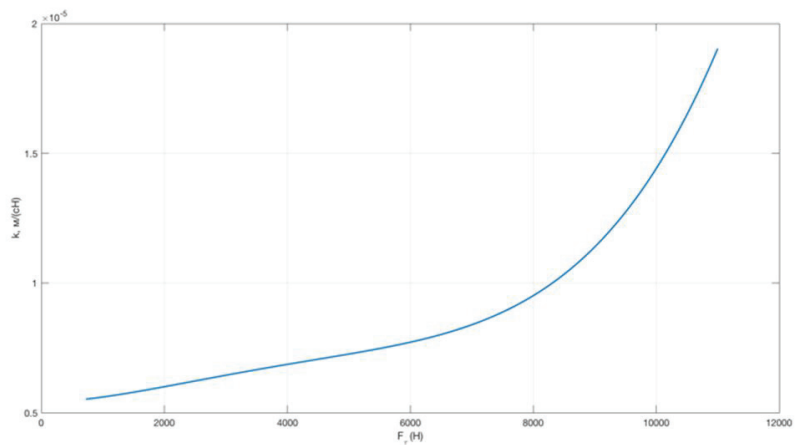

Fig. 2. Dependence of the coefficient of influence k on the tangential load on the wheel
Thus, this value can be used as a diagnostic criterion for the pre-sliding state. For its identification, it is proposed to use the spectral analysis of the vertical and horizontal oscillations of the wheel.

Consider a simplified "wheel-rail" system (see Figure 3).

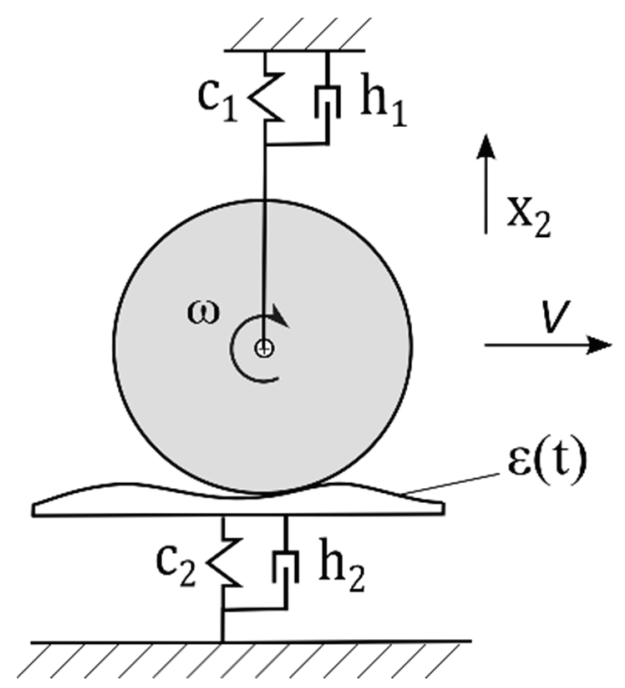

Fig. 3. The "wheel-rail" system.

Here $\varepsilon$ characterizes the unevenness of the railway track and acts as a source of vertical disturbances. The properties of $\varepsilon$ are considered in detail in [17-20]. Without going into detail on this issue, we note that $\varepsilon$ can be considered as white noise. The mathematical model describing the vertical oscillations in the given system will have the form:

$$
m \ddot{x}_{2}+\left(h_{1}+h_{2}\right) \dot{x}_{2}+\left(c_{1}+c_{2}\right) x_{2}=c_{2} \varepsilon(t)+h_{2} \dot{\varepsilon}(t)
$$

The mathematical model describing the horizontal oscillations in the operator form will have the form:

$$
\tilde{v}(p)=W_{v n}(p)\left(c_{1} x_{2}(p)+p h_{1} x_{2}(p)\right)
$$

It is easy to see that the transfer function of such a system along the $\varepsilon \rightarrow \tilde{v}$ channel can be written as follows:

$$
W_{\varepsilon v}(p)=\mathrm{W}_{v \tau} \frac{\left(c_{2}+h_{2} p\right)\left(c_{1}+h_{1} p\right)}{m p^{2}+\left(h_{1}+h_{2}\right) p+\left(c_{1}+c_{2}\right)^{\prime}}
$$

but along the $\varepsilon \rightarrow x_{2}$ channel:

$$
W_{\varepsilon x}(p)=\frac{c_{2}+h_{2} p}{m p^{2}+\left(h_{1}+h_{2}\right) p+\left(c_{1}+c_{2}\right)} .
$$

The results of numerical simulation show that the main intensity of the wheel oscillations is concentrated in a narrow frequency range determined by the system parameters $\left(\omega_{\max }=51 \mathrm{~Hz}\right.$ for typical railroad and wheel parameters). See Figure 4. 


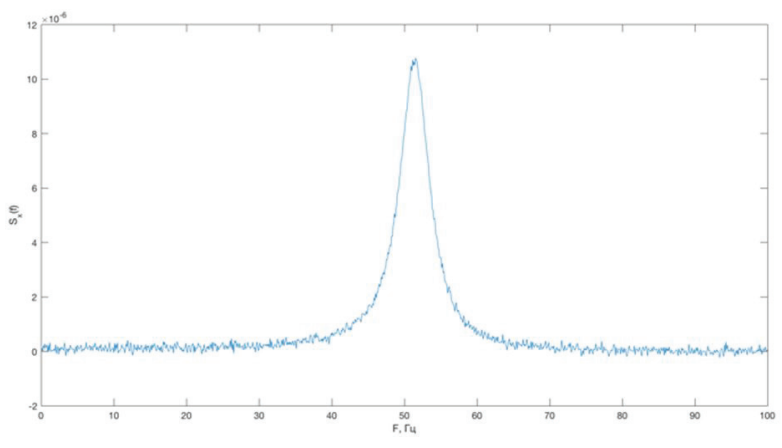

a

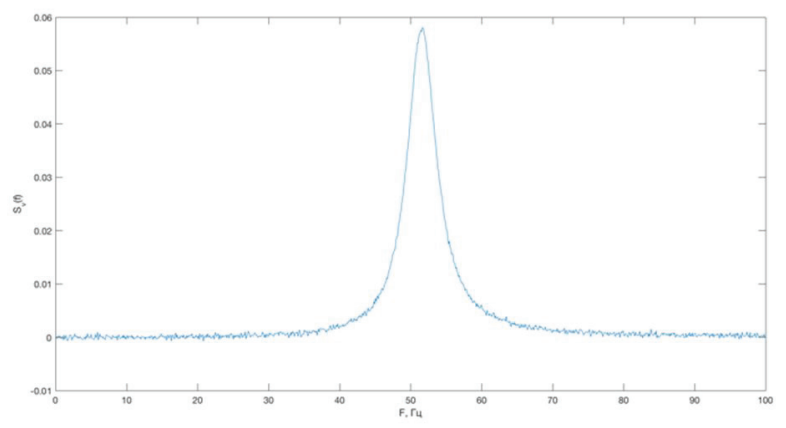

$\mathrm{b}$

Fig. 4. The spectral density of oscillations: a - vertical, b horizontal.

Based on the results of the simulation, it can be concluded that the main intensity of the wheel oscillations is concentrated in the frequency range $f=51$ $\mathrm{Hz}$ and is determined mainly by the properties of track stiffness and dissipation, and not by the elasticdissipative properties of the suspension. In addition, it can be noted that the relationship between the vertical oscillations of the centre of the wheel and horizontal velocity fluctuations is mainly at the frequency of vertical vibrations.

Also, based on the results of the simulation, it can be clearly seen that the spectral density of velocity oscillations at a frequency $\mathrm{f}=51 \mathrm{~Hz}$ depends significantly on the value of the tangential load $F_{\tau}$ and increases significantly as it approaches the pre-sliding state.

The value of the spectral density at the considered frequency is practically independent of the frequency properties of $\mathrm{W}_{v \tau}$ and is mainly determined by the influence coefficient $k$. Indeed, analysing the frequency characteristics of $W_{v \tau}$, it is easy to see that in the given frequency range, $\mathrm{W}_{v \tau} \approx k$. Using this relation, as well as formulas (4) and (5), one can obtain an expression for the spectral densities of the observed signals:

$$
\begin{gathered}
S_{v}(\omega)=k^{2}\left|\frac{\left(c_{2}+h_{2} j \omega\right)\left(c_{1}+h, j \omega\right)}{m(j \omega)^{2}+\left(h_{1}+h_{2}\right) j \omega+\left(c_{1}+c_{2}\right)}\right| S_{\varepsilon}(\omega) \\
S_{x}(\omega)=\left|\frac{c_{2}+h_{2} j \omega}{m(j \omega)^{2}+\left(h_{1}+h_{2}\right) j \omega+\left(c_{1}+c_{2}\right)}\right| S_{\varepsilon}(\omega)
\end{gathered}
$$

The ratio of the relations obtained is invariant to an external unobservable disturbance:

$$
\frac{S_{v}(\omega)}{S_{x}(\omega)}=k^{2}\left(c_{1}^{2}+h_{1}^{2} \omega^{2}\right)
$$

and allows the identification of the parameter $k$ with known suspension parameters $\left(c_{1}\right.$ and $\left.h_{1}\right)$ at a specified frequency $\omega_{\max }$ :

$$
k=\sqrt{\frac{S_{v}\left(\omega_{\max }\right)}{S_{x}\left(\omega_{\max }\right)\left(c_{1}^{2}+h_{1}^{2} \omega_{\max }^{2}\right)}} .
$$

As the value of the parameter $k$ is related to the state of the contact and increases as it approaches the pre-sliding state, this value can be used as a diagnostic criterion for the pre-sliding state. Thus, the task of dynamic diagnostics can be solved by observing the power ratio of the vertical and horizontal oscillations of the wheel to detect the critical value $k_{\max }$ at which a loss of adhesion traction occurs.

\section{Conclusion}

A mathematical model of the "wheel-rail" system is proposed, which considers the deformation processes occurring in the wheel. Numerical simulation of the system made it possible to find out that the loss of adhesion traction occurs gradually as the tangential load increases. In this case, structural changes occur in the contact leading to a change in the deformation diagram of the wheel. This influences the effect of so-called deformation sliding, which in turn affects the relationship of small wheel vibrations in the horizontal and vertical directions. Formula (6) allows, by observing the ratio of spectral densities, to identify the coefficient linking these oscillations $k$. The information on the magnitude of this coefficient makes it possible to determine the degree of proximity of the system to the pre-sliding state.

Practical implementation of this method does not require the use of complex, expensive technical solutions, and, therefore, may be of practical interest.

\section{References}

1. R.V. Chernov, Modern anti-skid protection on electric locomotives (The Urals Electromech. Institute of Engineering, Transp., 1977)

2. V.V. Deev, G.A. Ilyin, GS Afonin. Draft of trains (Transp., Moscow, 1987)

3. V.K. Garg, R.V. Dukkipati, Dynamics of rolling stock (Transp., Moscow, 1988)

4. F.T. Barweli, R.G. Woolacott, Proc. Instit. of Mechanical Engineers, 177, 145 (1963)

5. A.Ya. Kogan, Vniizht bulletin. 5, 33 (2004)

6. M.V. Chuveyko, S.V. Nosachev, Bulletin of the Don state tech. univ., 78, 127 (2014)

7. M.V. Chuveyko, S.V. Nosachev, Modern trends of development of science and tech., 2-3, 112 (2016) 
8. N. Matsui, K. Yokose, Railway Technical Research Institute, 3 (1966)

9. J.J. Kalker, Vehicle System Dynamics, 5, 317 - 358 (1979)

10. J.J. Kalker, In The General Problem of Rolling Contact. Transactions of American Society of Mechanical Engineers, 40, 77-92 (1980)

11. F.T. Barweli, Woolacott R.G. Proceedings Institution of Mechanical Engineers, 177, 145 - 160 (1963)

12. G.S. Itami, The study of Friction - Creep Phenomenon of adhesion between Steel Wheels and Rail (General Motors Institute, 1968)

13. K. Johnson, Mechanics of contact interaction. (Moscow, The World, 1989)

14. A.Yu. Ishlinsky, I.V. Kragelsky, Technical Physics, 45, 276 - 282 (1944)

15. L. Bureau, Comptes Rendus de l'Académie des Sciences, 2, 695-796 (2001)

16. V.L. Veits, M.S. Bundur, V.E. Hitrik, Journal of Friction and Wear, 6, 653 - 660 (1985)

17. M.F.Verigo, A.Ya. Kogan, Interaction of the track and rolling stock. (Transp., Moscow, 1986)

18. S.V. Vershinsky, V.N. Danilov, I.I. Chelnokov, The dynamics of the car. (Transp., Moscow, 1978)

19. C. Esveld, A. Kok, The Railways of the World, 12 (2000)

20. V.F. Ushkalov, L.M. Reznikov, L.P. Boyarintseva, Vniizht bulletin, 6 (1986) 